\title{
Eficacia del entretenimiento de la musculatura del suelo pélvico en incontinencia urinaria femenina
}

\author{
Efficacy of training pelvic floor musculature in female \\ urinary incontinence
}

\author{
B. González Sánchez ${ }^{1}$, J. Rodríguez-Mansilla ${ }^{1}$, A. de Toro García ${ }^{2}$, \\ M.V. González López-Arza ${ }^{1}$
}

\section{RESUMEN}

Se define incontinencia urinaria "como la pérdida involuntaria de orina, que condiciona un problema social e higiénico". Existen gran variedad de técnicas para el tratamiento de la incontinencia urinaria.

El objetivo es conocer la efectividad clínica de los diversos métodos utilizados para el entrenamiento de la musculatura del suelo pélvico como tratamiento de la incontinencia urinaria femenina de esfuerzo o mixta y su contribución a la mejora de la calidad de vida de estas pacientes.

Para ello hemos realizado una revisión sistemática de ensayos clínicos controlados. Tras analizar los resultados podemos concluir que el tratamiento de la incontinencia urinaria de esfuerzo o mixta mediante ejercicios de entrenamiento de la musculatura del suelo pélvico contribuye de manera positiva a mejorar los síntomas, la fuerza de la musculatura del suelo pélvico y la calidad de vida de las pacientes afectas.

Palabras clave. Ejercicio físico. Incontinencia urinaria femenina. Suelo pélvico. Músculo. Entrenamiento.

\begin{abstract}
Urinary incontinence "as the involuntary loss of urine, which conditions a social and hygienic problem" is defined. There are a variety of techniques for the treatment of urinary incontinence.

The aim is to determine the clinical effectiveness of different methods used to train the musculature of the pelvic floor as treatment for female urinary incontinence, both stress and mixed, and its contribution to improving the quality of life of these patients.

For this purpose we performed a systematic review of controlled clinical trials. After analysing the results, we conclude that the treatment of stress or mixed urinary incontinence through training exercises for the musculature of the pelvic floor contributes positively to improving symptoms and strengthening the muscles of the pelvic floor and to the quality of life of affected patients.
\end{abstract}

Key words. Physical exercise. Female urinary incontinence. Pelvic floor. Muscle. Training.
1. Universidad de Extremadura. Facultad de Medicina. Departamento de Terapéutica MédicoQuirúrgica. Badajoz (España).

2. IMC Clínica Fisioterapia. Cáceres (España).

Recepción: 16 de julio de 2014

Aceptación provisional: 1 de septiembre de 2014

Aceptación definitiva: 29 de septiembre de 2014

\section{Correspondencia:}

Blanca González Sánchez

Facultad de Medicina

Universidad de Extremadura

Badajoz (España)

E-mail: bgs2313@hotmail.com

\section{Conflicto de intereses}

Los autores declaran no tener ningún conflicto de intereses 


\section{INTRODUCCIÓN}

Se define incontinencia urinaria (IU) "como la perdida involuntaria de orina, que condiciona un problema social e higiénico y que será condición indispensable para que se produzca el escape, que la presión vesical supere a la uretral"1.

Está considerada enfermedad por la OMS desde 1998, por su repercusión en la calidad de vida (CV), por su frecuencia y por su afectación psico-social ${ }^{2}$.

Las cifras de prevalencia que aparecen en los diferentes estudios son muy variables, dependiendo de distintos factores como concepto de incontinencia, método de estudio, nivel asistencial estudiado y otras características de las muestras ${ }^{3}$, además, se suma que existe un gran número de personas difíciles de diagnosticar ya que tienden a ocultarlo o lo asumen como un problema propio de la edad ${ }^{4}$. En general, se observa que afecta más a la mujer que al hombre y que aumenta con la edad ${ }^{5,6}$. Según un estudio realizado por la Asociación Española de Enfermería en Urología6 la prevalencia de la IU en el sexo femenino es del 20 al $30 \%$ en las mujeres de edad media y del 30 al $50 \%$ en mujeres mayores de 65 años.

Atendiendo a los criterios sintomáti$\cos ^{3}$ y por orden de prevalencia los tipos de incontinencia urinaria que podemos encontrar son:

I.U. de Esfuerzo (IUE): se define como la pérdida involuntaria de orina asociada a un esfuerzo físico que provoca un aumento de la presión abdominal ${ }^{3}$. Se produce cuando hay un aumento súbito de la presión intraabdominal, el esfínter no es capaz de soportarla y se produce el escape ${ }^{2}$. Otra causa por la que se produce es por descenso de la posición normal del ángulo uretrovesical posterior ${ }^{7}$. Es la más frecuente en mujeres ${ }^{4}$.

I.U. Mixta (IUM): es la percepción de pérdida involuntaria de orina asociada tanto a urgencia como al esfuerzo ${ }^{3}$. La única forma de identificarla es mediante un estudio urodinámico ${ }^{7}$. Es más frecuente en mujeres premenopáusicas ${ }^{3}$.
I.U. de Urgencia (IUU): es la incapacidad para contener la orina el tiempo suficiente para ir al baño, siendo la más frecuente en personas mayores ${ }^{4}$. Se produce por contracciones involuntarias del detrusor, que aparecen mientras el paciente trata de inhibir la micción ${ }^{7}$. Cuando estas contracciones involuntarias se ponen de manifiesto en un estudio urodinámico se denomina "detrusor hiperactivo" y cuya causa puede ser una enfermedad neurológica $^{3}$.

I.U. por rebosamiento: frecuente en varones por obstrucción prostática. Se produce en presencia de retención y residuos permanentes por hipoactividad del detrusor. La vejiga está sobredistendida y se producen escapes inadvertidos de escasa cantidad pero casi constantes ${ }^{7}$.

I.U. inconsciente: es la pérdida involuntaria de orina sin deseo miccional e independientemente de cualquier aumento de la presión abdominal ${ }^{3}$.

La literatura médica indica que existen una gran variedad de técnicas para el tratamiento de la incontinencia urinaria y éstas deberán ajustarse más al tipo de paciente que al tipo de incontinencia que padezca. Entre las diferentes técnicas de tratamiento existe el tratamiento conservador, farmacológico o quirúrgico. El tratamiento conservador es considerado en la actualidad como un elemento esencial en el abordaje terapéutico. Generalmente su aplicación es previa y/o simultánea a otras medidas farmacológicas o quirúrgi$\operatorname{cas}^{3}$ e incluye las siguientes medidas generales: ingesta moderada de líquidos (1-1,5 l/día), evitar cafeína y alcohol ${ }^{7}$ y fortalecer la musculatura pélvica incluyendo técnicas de BF (bio-feedback) ${ }^{3,7}$.

El empleo de fármacos, en el tratamiento de la incontinencia urinaria, se basa en la existencia de neurorreceptores colinérgicos, beta adrenérgicos y alfa adrenérgicos, que producen contracción o relajación vesicouretral en función del fármaco administrado ${ }^{7}$.

El tratamiento quirúrgico es el adecuado en los pacientes en los que el tratamiento conservador ha fracasado o 
en aquellas personas con incontinencia urinaria de esfuerzo moderada-severa. Su propósito es incrementar la resistencia uretral para evitar el escape de orina por la uretra durante los aumentos de la presión intraabdominal, preservando el vaciamiento vesical completo a baja presión $^{3}$. El propósito de las técnicas quirúrgicas es colocar el cuello vesical y la uretra proximal en una posición intraabdominal y lograr una compresión adecuada de la unidad esfinteriana. Las técnicas más utilizadas hasta el momento eran la colposuspensión de Burch o la técnica de Marshall-Marchetti-Krantz ${ }^{7}$, sin embargo ambas se han visto remplazadas con la aparición de las cintas suburetrales. Ulmsten y Petros ${ }^{8,9}$ fueron los primeros en describir la técnica con cinta vaginal libre de tensión y desde entonces se han convertido en unas de la técnicas quirúrgicas más utilizadas. Se ha demostrado que la utilización de dichas cintas posee una tasa de éxito mayor que otras técnicas quirúrgicas y que el tiempo de recuperación tras su colocación es menor que con la cirugía convencional ${ }^{8,9}$.

La evidencia médica indica que el tratamiento quirúrgico es considerado como el más efectivo para la resolución de la IUE, sin embargo los efectos secundarios postoperatorios han favorecido la utilización de otros métodos menos agresivos para el tratamiento de la IUE siendo uno de los más utilizados actualmente la rehabilitación de la musculatura del suelo pélvico mediante diversos métodos de entrenamiento ${ }^{10}$.

Por todo lo anteriormente expuesto, el objetivo de esta revisión es conocer por medio de los estudios más recientes, la efectividad clínica de los diversos métodos utilizados para el entrenamiento de la musculatura del suelo pélvico como tratamiento de la incontinencia urinaria femenina de esfuerzo o mixta y su contribución a la mejora de la calidad de vida de estos pacientes, contribuyendo de esta manera a la difusión de esta patología y abriendo puertas a tratamientos no invasivos de la misma.

\section{MATERIAL Y MÉTODOS}

Revisión sistemática de ensayos clínicos controlados. Se recogen artículos en inglés o español publicados desde enero del 2003 hasta marzo del 2013. Todos dirigidos a pacientes femeninas que presenten una incontinencia urinaria de esfuerzo o mixta y cuyo método de tratamiento sea el entrenamiento de la musculatura del suelo pélvico.

Los criterios de exclusión son: artículos científicos que aborden la incontinencia urinaria desde otro punto de vista fisioterápico que no sea el entrenamiento de la musculatura del suelo pélvico; aquellos en los cuales las pacientes presenten incontinencia urinaria que no sea de esfuerzo o mixta o asociada a otra patología de base tal como enfermedad neurológica; prolapso urogenital, menos de 3 meses del parto o como consecuencia de una cirugía, aquellos que no se encuentren en inglés o español y aquellos que no se correspondan con la tipología de estudios incluidos (ensayos clínicos).

La calidad metodológica de los estudios incluidos se realizó utilizando la escala PEDro (Physiotherapy Evidence Databa$s e)^{11}$, que indica la calidad de los ensayos. Se compone de 11 criterios con respuestas "SI" (S) O "No" (N) y un rango de puntuación total que varía de 0 a 10 según tengan baja o excelente calidad metodológica. Los criterios del 2 al 9 pretenden justificar si el estudio tiene o no suficiente validez interna, con los criterios 10 y 11 se prueba si la información estadística es suficiente para interpretar los resultados y la validez externa va relacionada con el primer criterio. Éste último es adicional y no se utiliza para el cálculo de la puntuación es por ello que la máxima puntuación que se puede obtener es de 10 y no de 11.

Los 11 criterios que se evalúan con la escala PEDro son:

1. Especificación de los criterios de elegibilidad; 2. Asignación aleatoria; 3. Asignación ocultada, 4. Base comparable; 5 . Paciente "enmascarado"; 6. Terapeuta "enmascarado"; 7. Evaluador "enmascarado"; 8. Seguimiento del sujeto (al menos $85 \%$ de seguimiento); 9. Análisis del tipo intención 


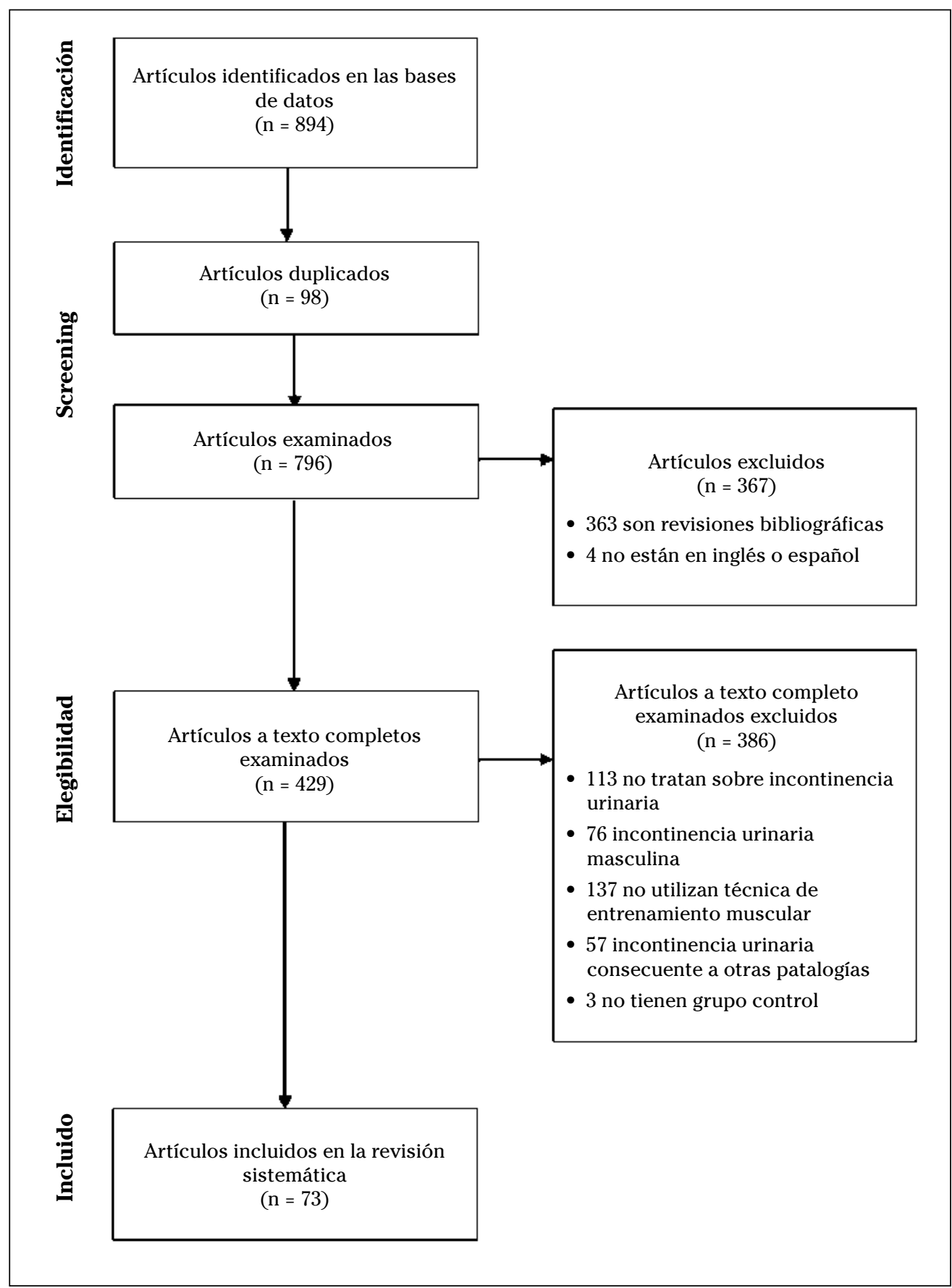

Figura 1. Proceso de selección y búsqueda de los estudios. 
de tratar; 10. Comparaciones estadísticas entre los grupos; 11. Medida de variabilidad y punto de medidas.

Los valores obtenidos en la escala se considera de: alta calidad, si la puntuación obtenida es mayor a 5 (6-8: bueno, 9-10 excelente); calidad moderada, si la puntuación es de 4 o 5 (estudio justo) y baja calidad, si la puntuación es menor de 4 (estudio pobre).

\section{Estrategia de búsqueda}

La búsqueda se realizó en marzo de 2013, revisando los estudios más recientes (últimos 10 años) y en los idiomas de español e inglés.

Se utilizaron las bases de datos electrónicas MEDLINE, Dialnet, Cochrane Library Plus, y "The Physiotherapy Evidence Database" PEDro. Se utilizaron las combinaciones de los términos Mesh "physical exercise", "urinary incontinence", "pelvic floor", "muscle" y "training", utilizando el operador booleano AND.

Dos revisores independientes realizaron una crítica de los artículos encontrados. En caso de desacuerdo, se empleó una puesta en común de los resultados y se llegó a un consenso entre ambos.

Como norma general, se realizó una preselección de las publicaciones considerando su adecuación a la temática propuesta en esta revisión.

Se estableció una selección de los artículos y se procedió a la lectura de su resumen o abstract, excluyendo aquellos artículos que no cumplían con los criterios de inclusión anteriormente reflejados. Las publicaciones que superaron los criterios anteriores fueron sometidas a su lectura completa para el posterior análisis e inclusión en esta revisión.

\section{RESULTADOS}

De los 894 artículos encontrados en el proceso de búsqueda se seleccionaron 43 en base a los criterios de inclusión y exclusión expuestos anteriormente.

El proceso de búsqueda y selección de los estudios relevantes se resume en la figura 1.

\section{DESCRIPCIÓN DE LOS ESTUDIOS}

Las principales características de los estudios que se incluyen en esta revisión quedan reflejadas en la tabla 1 .

La muestra de estudios es variable. $\mathrm{La}$ mayor muestra, $\mathrm{N}=245$ es la del trabajo de Liebergall-Wischnitzer y $\operatorname{col}^{33}$ y la menor, $\mathrm{N}=11$, la del trabajo realizado por Donahoe Fillmore $\mathrm{E}$ y col${ }^{20}$.

El objetivo de todos los trabajos analizados es evaluar la eficacia del entrenamiento de la musculatura del suelo pélvico como método de tratamiento de la IUE o IUM, mediante un grupo experimental y comparándolo con otras técnicas de tratamiento o grupo control.

La duración de las intervenciones es muy heterogénea y varía entre 3 semanas ${ }^{30}$ y un año ${ }^{15}$, siendo lo más común un tratamiento de 12 semanas ${ }^{23-25,31-35,49-51}$ de duración. En uno de los trabajos ${ }^{47}$ no se especifica la duración de la intervención.

En todos los estudios analizados se ha hecho una evaluación pre tratamiento y otra evaluación inmediatamente al terminar el mismo. Algunos realizan evaluaciones de seguimiento una vez concluida la intervención, a las 6 semanas $^{23,36}$, a $3^{28,30,48}$ y 7 meses $^{31} \mathrm{y}$ al año ${ }^{13,15,29}$ de la misma.

A la hora de analizar los métodos de evaluación empleados, la heterogeneidad aumenta aún más, ya que dependiendo del tipo de ejercicio realizado han sido utilizados unos u otros. Los test más utilizados por la mayoría de los trabajos son: Pad test $^{12,21,24,32-34,48}$, I-QoL (Incontinence Quality of Life Questionnaire) $)^{17,32-36,41,54}$, KHQ (King Health Questionnaire) $)^{17,19,25,36-38}$ y EVA (Escala Visual Analógica) $)^{10,12,21,43}$, además de la fuerza de la musculatura del suelo pélvico, medida en la mayoría de los casos mediante la Escala Oxford ${ }^{18,22,31,38}$. Donahoe Fillmore y $\mathrm{col}^{20}$ utilizan también el test UDI 6 SF para medir los resultados obtenidos. POPUISQ (Pelvic Organ Prolapse/Urinary Incontinence Sexual Questionnaire) tan solo fue utilizada en el trabajo de Liebergall-Wischnitzer y col${ }^{34}$. Algunos autores ${ }^{14,18,22}$ incluyen como medida de evaluación la opinión del paciente tras el tratamiento, a pesar de ser una medida subjetiva. La perineometría ${ }^{10,32,36,41,51,52}$ es la prueba objetiva más utilizada en todos los trabajos analizados. 


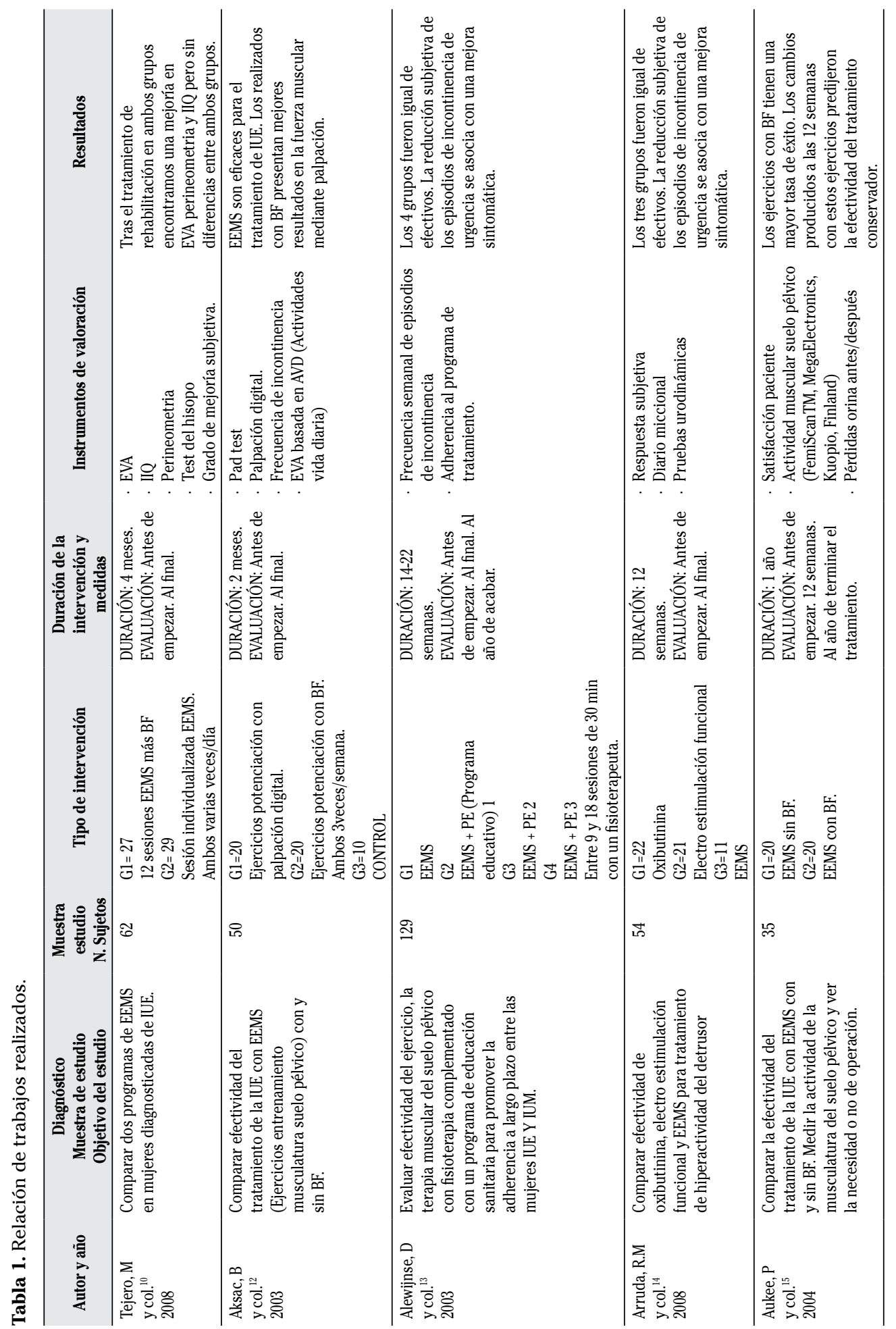




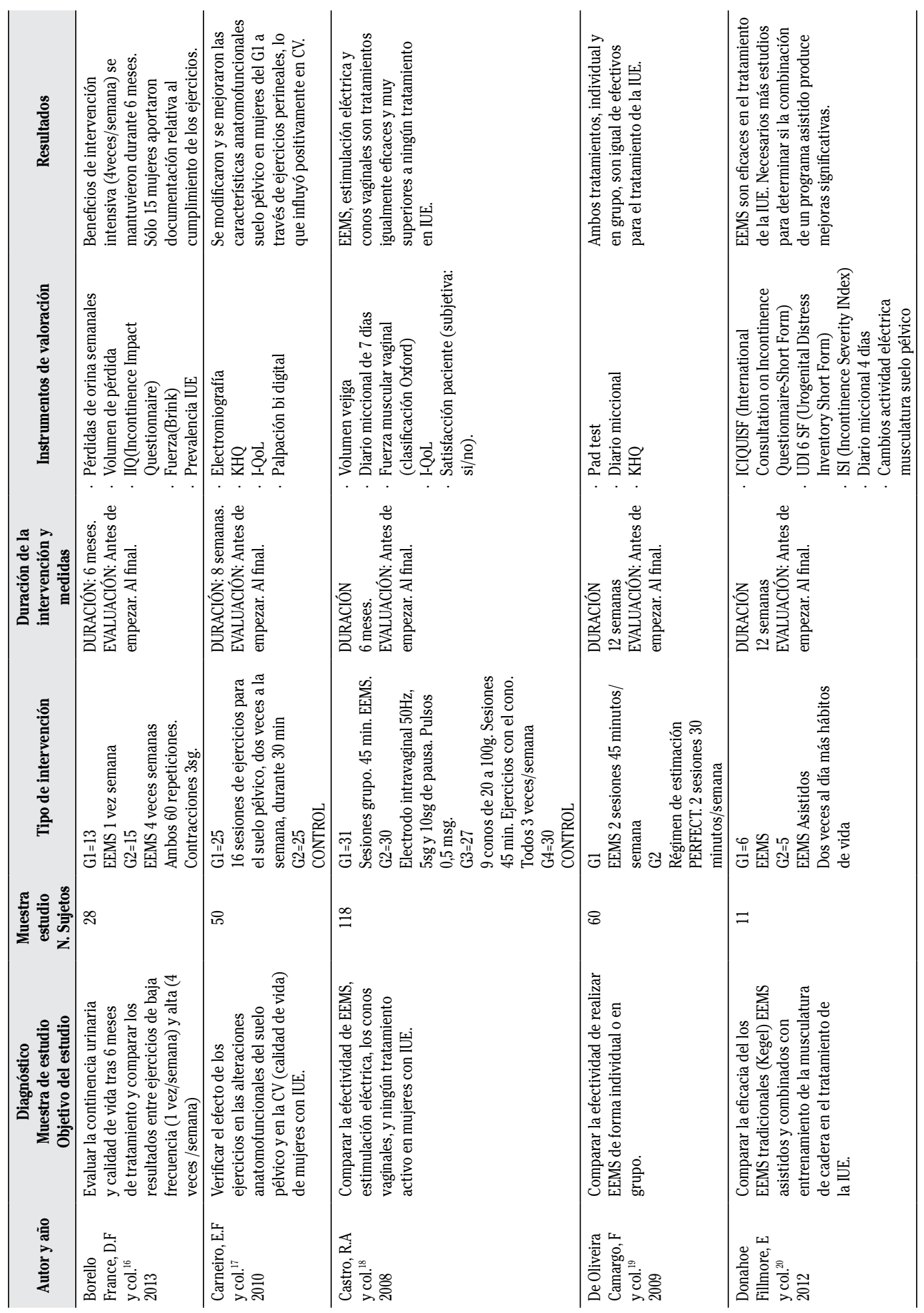




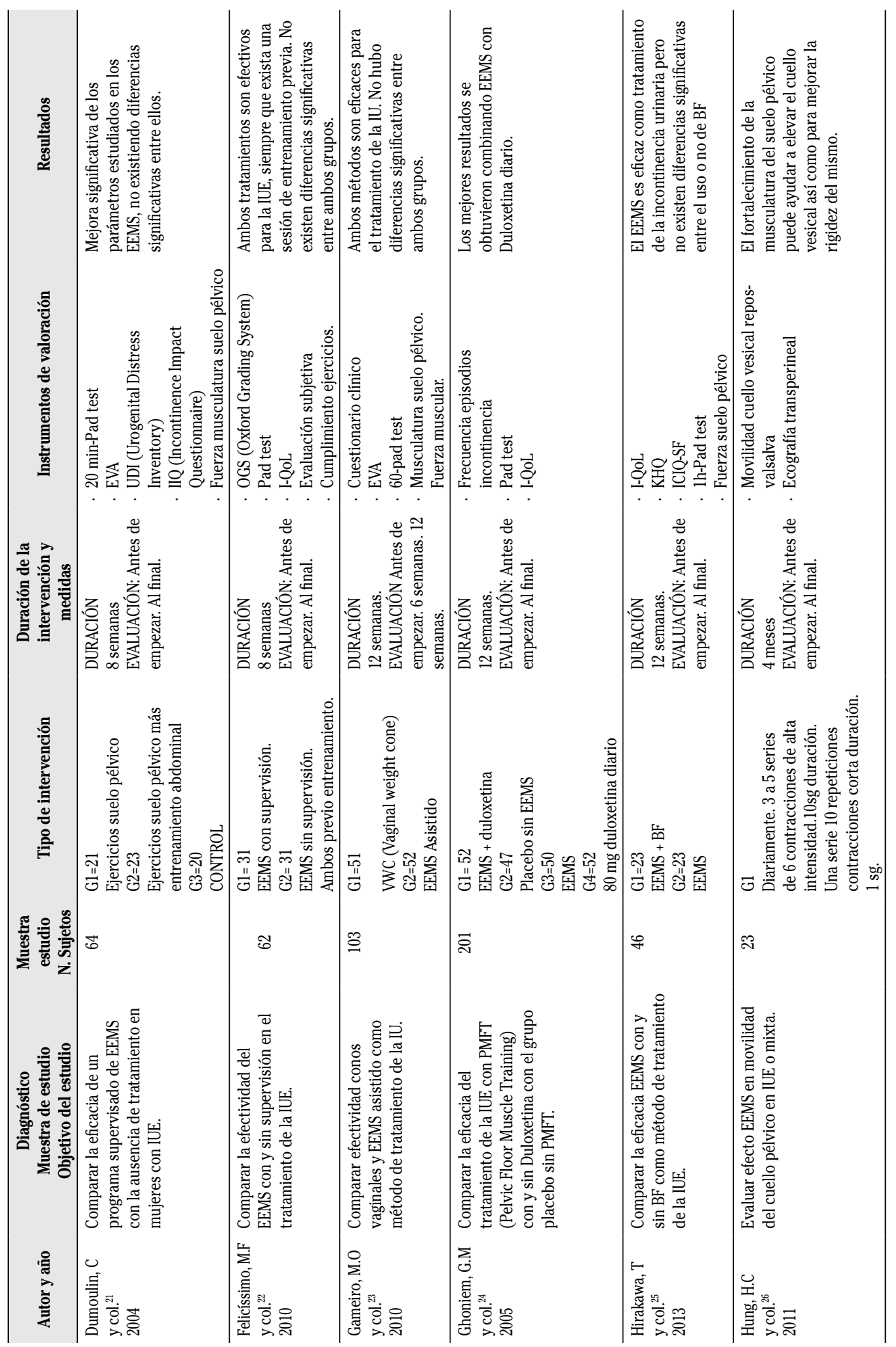




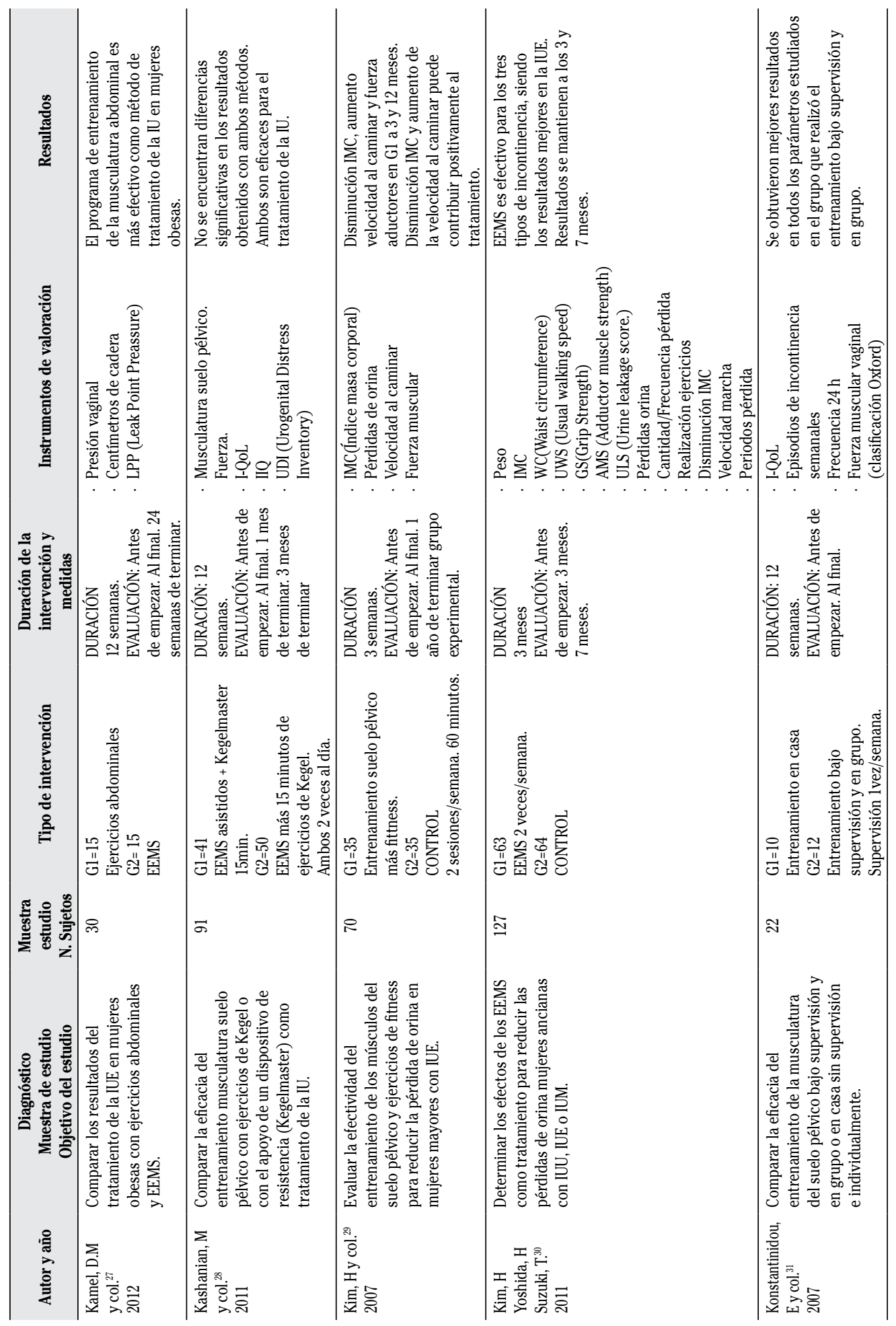




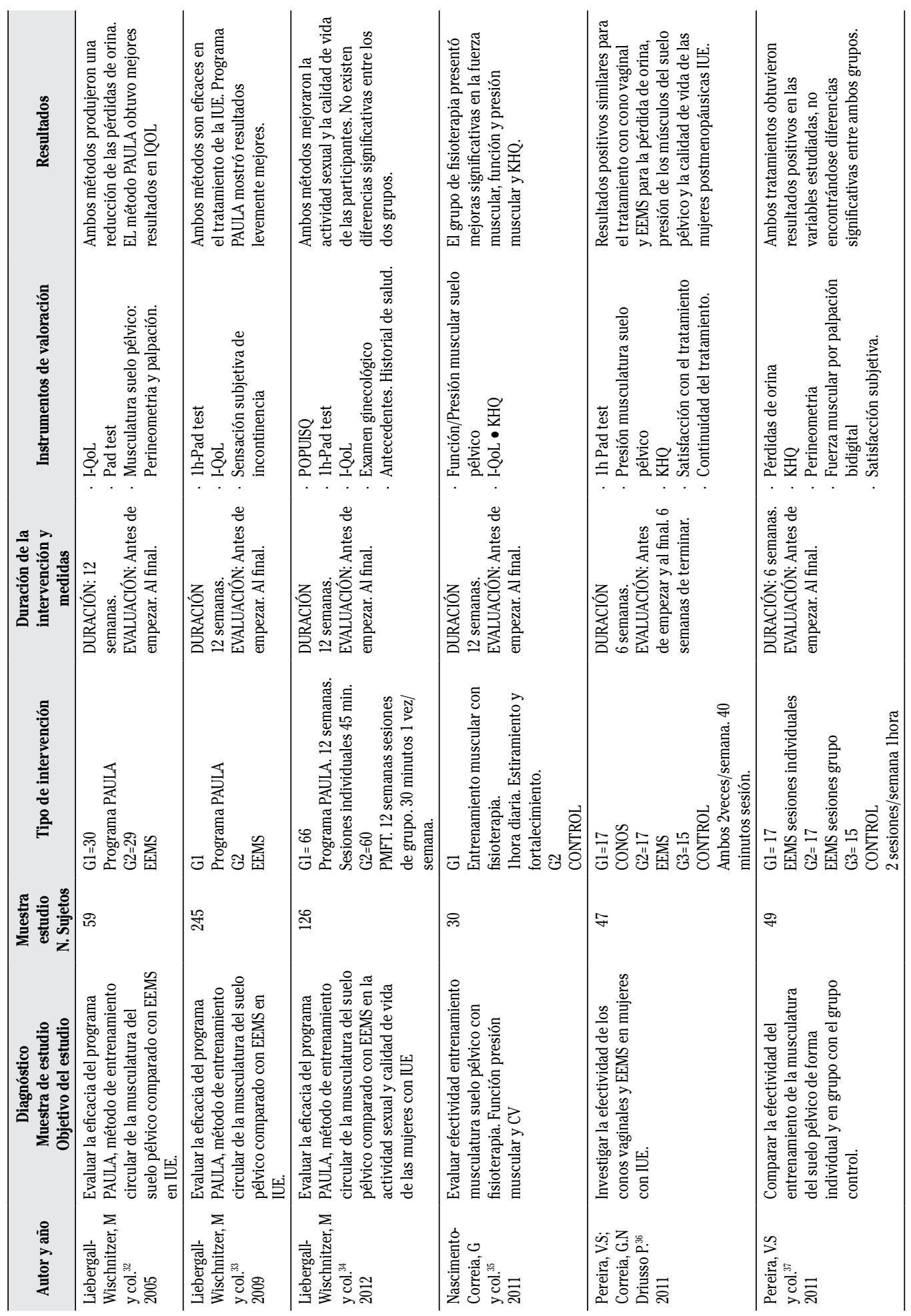




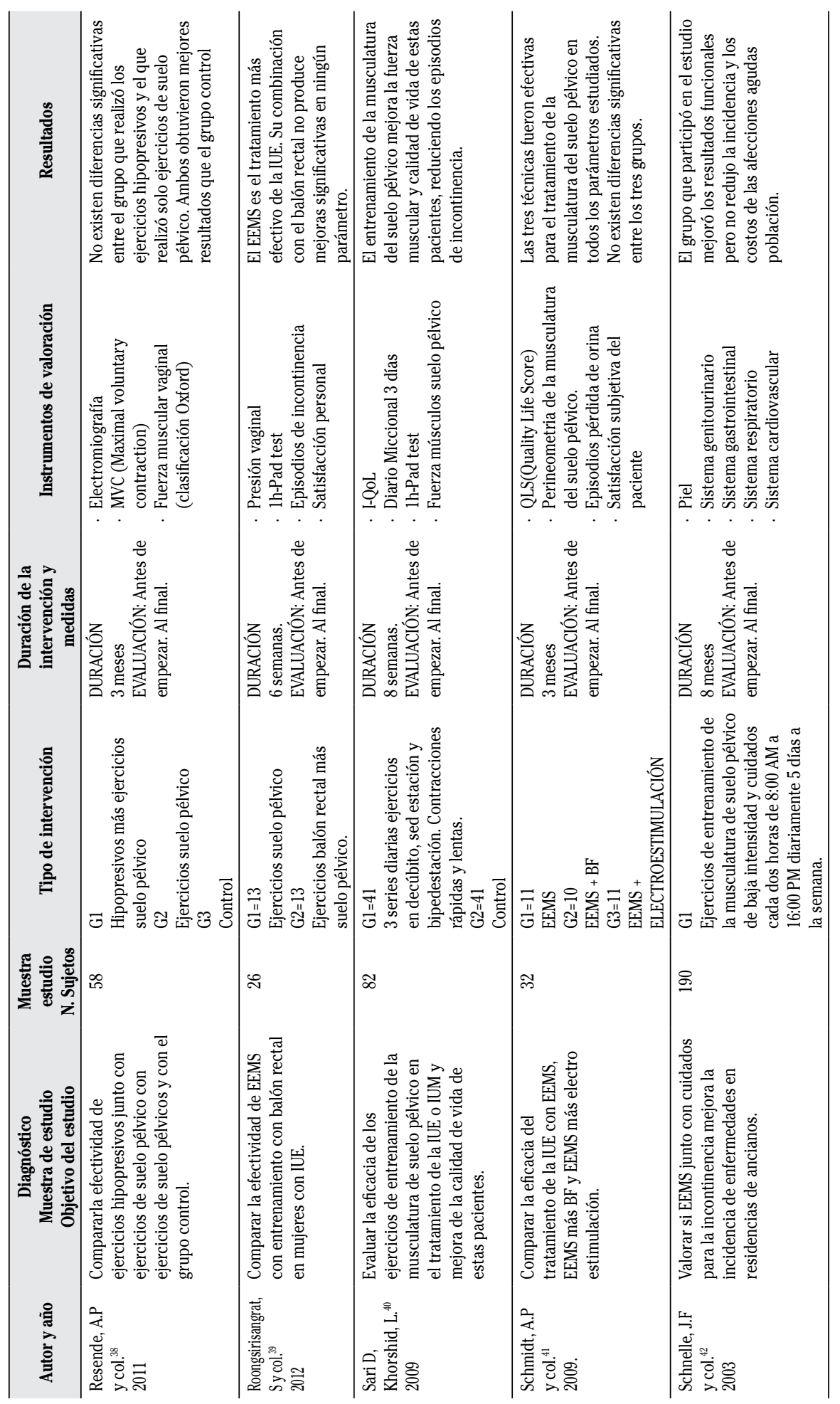




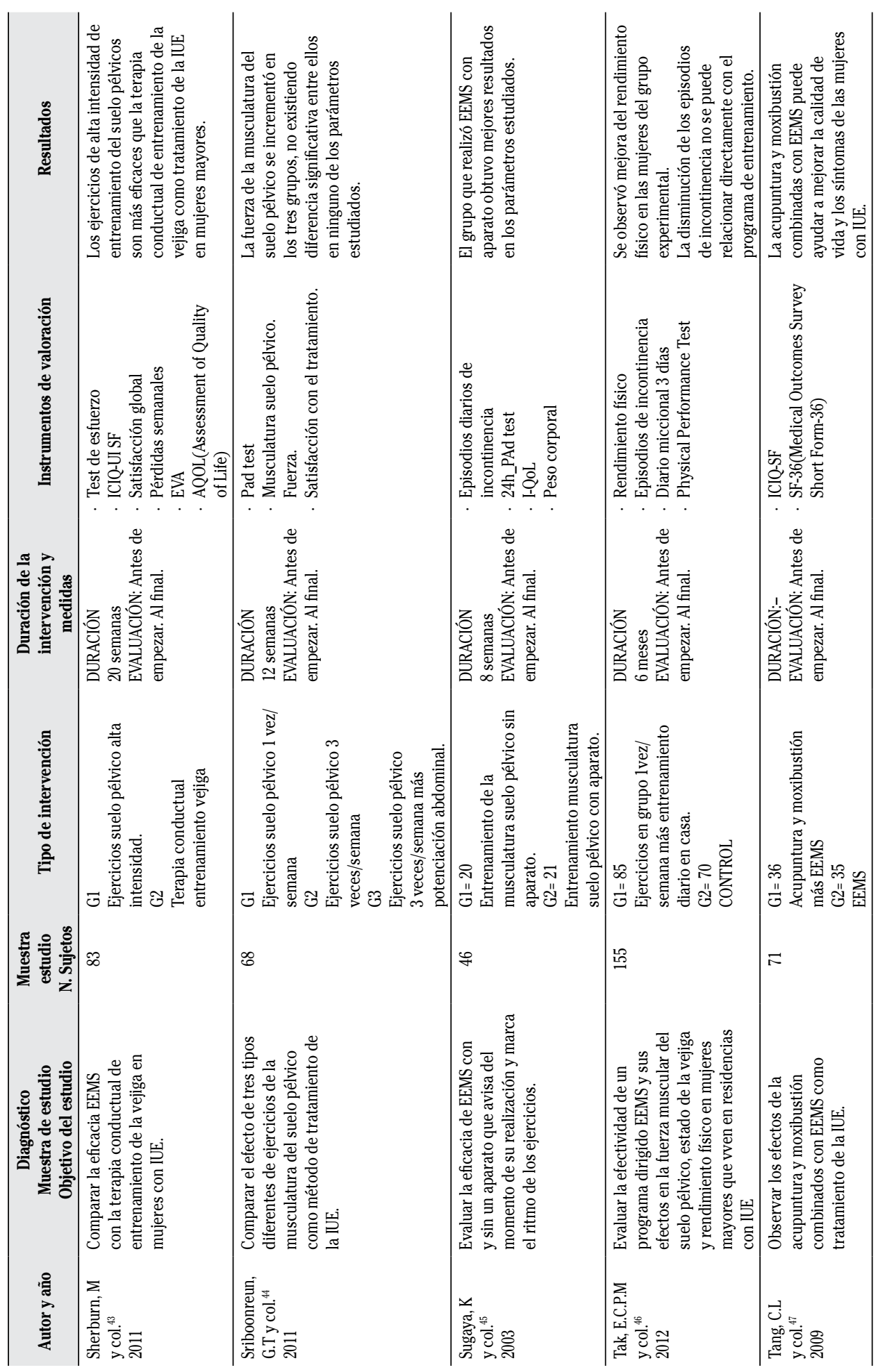




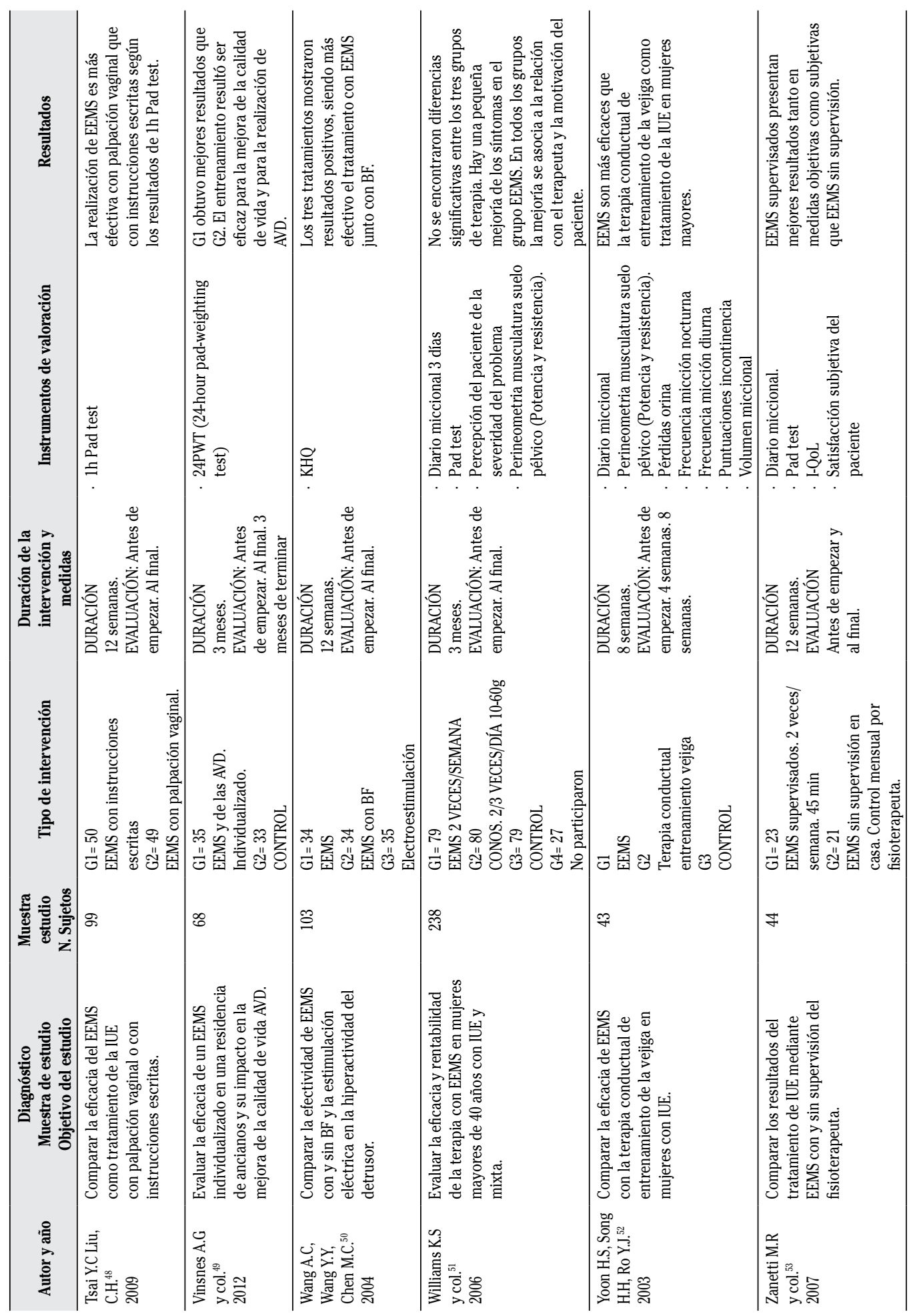


En todos los trabajos analizados existe un grupo en el que el tratamiento ha consistido en ejercicios de entrenamiento de la musculatura del suelo pélvico. Las diferencias entre los trabajos son el tipo de ejercicio realizado como entrenamiento de dicha musculatura, la presencia o no de grupo control y los diferentes métodos con los que han comparado el entrenamiento practicado por el grupo experimental. Todos los que incluyen un grupo control tienen en común que este grupo no ha recibido ningún tipo de tratamiento, excluyendo por tanto todos los que han recibido algún tipo de tratamiento placebo.

Aksac y col $^{12}$ comparan ejercicios de entrenamiento de la musculatura del suelo pélvico con y sin palpación digital con el grupo control, realizando ambos grupos experimentales 3 sesiones de ejercicios semanales. En otros trabajos se compara la eficacia del entrenamiento de la musculatura del suelo pélvico frente al uso de fármacos, como la oxibutinina ${ }^{14}$ o duloxetina ${ }^{24}$.

La electroterapia ${ }^{18,41,50}$, mediante electrodo vaginal y los conos vaginales ${ }^{15,51}$ son otros de los métodos utilizados en los trabajos analizados para comparar su eficacia frente a los ejercicios de entrenamiento de la musculatura.

Liebergall-Wischnitzer y col $^{32-34}$ comparan en todos sus trabajos la realización de ejercicios de entrenamiento de dicha musculatura del suelo pélvico con el programa PAULA, un método de entrenamiento circular de dicha musculatura. Los programas educativos ${ }^{13}$ y la terapia conductual ${ }^{43,52}$ son otros de los métodos estudiados.

El trabajo realizado por Tang y $\mathrm{col}^{47}$ es el único que compara los resultados obtenidos mediante la realización de ejercicios de suelo pélvico con los obtenidos con otras terapias alternativas como la acupuntura o moxibustión.

En todos los trabajos se observa una mejora significativa de los parámetros estudiados mediante el entrenamiento de la musculatura del suelo pélvico con ejercicios, concluyen que es un tratamiento eficaz en la IUE o IUM. Aksac y $\operatorname{col}^{12}$ y Aukee y col $^{15}$ además obtienen que los beneficios de este tratamiento aumentan cuando se acompaña el entrenamiento de la musculatura del suelo pélvico con BF.

Otros autores como Vinsnes y col $^{49}$ concluyen que los resultados obtenidos mejoran cuando se acompañan los ejercicios de suelo pélvico con pautas para la vida diaria.

El entrenamiento de la musculatura del suelo pélvico mediante ejercicios de potenciación de dicha musculatura produjo una disminución de los episodios de incontinencia ${ }^{13,14}$, un aumento de la fuerza de la musculatura del suelo pélvico ${ }^{35,40}$ y una mejora de la calidad de vida ${ }^{17}$, de las mujeres participantes. La investigación realizada por Liebergall-Wischnitzer y $\mathrm{col}^{34}$ afirma que los ejercicios de potenciación de la musculatura del suelo pélvico realizados mediante un método de entrenamiento circular contribuyen de forma positiva a mejorar la actividad sexual de las mujeres con IUE o IUM.

Los ejercicios más utilizados para potenciación de la musculatura del suelo pélvico son: potenciación de los músculos pubovaginales, puborectales y pubocoxígeos ${ }^{27}$, mediante ejercicios realizados simultáneamente al aumento de la presión abdominal ${ }^{21,29,38,44}$ o ejercicios de potenciación de la musculatura del suelo pélvico mediante ciclos de contracciones y reposo a diferente intensidad y frecuencia y/o acompañados de palpación digital ${ }^{12,48}$. En gran parte de los trabajos analizados los autores incluyen un entrenamiento previo del paciente en el cual toma conciencia de su musculatura y aprende a contraer o relajar dicha musculatura de forma voluntaria ${ }^{21}$.

\section{Evaluación de la calidad metodológica}

Las variables han sido evaluadas con calificaciones de "SÍ" o "NO", según la presencia o no del criterio estudiado, tal y como se refleja en la tabla 2 . Al poner la calificación "NO" no aseguramos que el estudio no tenga esa característica, sino que al revisar el artículo completo no se ha encontrado ese requisito reflejado en el texto.

Cuatro estudios ${ }^{14,20,31,53}$ obtuvieron una puntuación global de 3, pobre. La puntua- 
ción más alta fue la obtenida por el estudio de Hung y $\mathrm{col}^{26}$, siendo ésta de 8 , bueno. El resto de estudios evaluados obtuvieron puntuaciones entre 4 y 7 puntos.

Los artículos evaluados presentan una puntuación media de 7,22 puntos. Todos los artículos salvo el realizado por Zanetti MR y col $^{53}$ coinciden en la presencia del criterio 11 (medida de variabilidad y punto de medidas) y en la ausencia del criterio 6 (terapeuta enmascarado). Todos los trabajos presentan comparaciones estadísticas entre grupos (criterio 10) salvo el trabajo realizado por Kamel y $\mathrm{Col}^{27}$.

Solo en los estudios realizados por Ghoniem y coll ${ }^{23}$ y Sugaya y col ${ }^{44}$ está presente el criterio 5 (paciente enmascarado).

\section{DISCUSIÓN}

Aunque son muchos los estudios que hacen referencia a la incontinencia urinaria, en nuestra búsqueda inicial encontramos 894 artículos, el número de investigaciones sobre esta temática disminuyen cuando se centran en un tipo de incontinencia específica, en este caso IUE y IUM y sin estar asociada a otra patología.

Entre todas las técnicas utilizadas para el tratamiento de la IU el entrenamiento de la musculatura del suelo pélvico, el abordaje mediante ejercicios es una de las más comunes, combinada o no con otras terapias o métodos de tratamiento (Tabla 1). Sin embargo, debido a la heterogeneidad de ejercicios realizados, que abarcan desde ejercicios de entrenamiento de la musculatura del suelo pélvico realizados de forma aislada ${ }^{13-28,36-47,50-53}$, combinados con $\mathrm{BF}^{10,12,14,25,41}$, con programas educativos ${ }^{13} \mathrm{O}$ con acupuntura ${ }^{47}$ a ejercicios realizados con conos vaginales ${ }^{17,36,51}$ o ejercicios de entrenamiento mediante hipopresivos ${ }^{38}$, así como el número de variable de intervenciones realizadas, de 3 semanas $^{29}$ a 8 meses $^{42}$ resulta difícil afirmar qué tipo de ejercicio es el más apropiado para mejorar este problema.

Los artículos encontrados, concluyen que el tratamiento de la IUE o IUM mediante métodos de entrenamiento de la musculatura del suelo pélvico produce resultados positivos tanto en la reducción de los epi- sodios de incontinencia como en la mejora de la fuerza muscular a nivel del suelo pélvico, si bien los resultados mejoran cuando se combinan dichos ejercicios con otras técnicas como el biofeedback ${ }^{15}$, electrodos intravaginales ${ }^{18}, \operatorname{conos}^{18,51}$ o se realizan de manera asisitida ${ }^{31}$.

En este aspecto, en el estudio realizado por Aukee y col $^{15}$ la utilización del BF en combinación con dichos ejercicios mejora los resultados de los mismos, dichos resultados están en consonancia con los obtenidos por otros autores, como Wang y $\mathrm{col}^{50}$ los cuales en una investigación realizada en el mismo año obtuvieron mejores resultados en el grupo que combinó ejercicios de entrenamiento de la musculatura del suelo pélvico con BF. Sin embargo Tejero y $\operatorname{col}^{10} \mathrm{y}$ Schmidt y col $^{41}$ afirman no obtener diferencias significativas entre los grupos que realizaron ejercicios de entrenamiento de la musculatura del suelo pélvico con y sin BF.

Existe más homogeneidad de opiniones al comparar el entrenamiento de la musculatura del suelo pélvico mediante ejercicios con otras terapias. Los estudios analizados muestran resultados similares y positivos, en el tratamiento de la incontinencia urinaria con ejercicios de suelo pélvico, conos vaginales ${ }^{18,51}$ o electroestimulación ${ }^{41,50}$ mediante electrodo vaginal, destacando en estos estudios la efectividad de dichos métodos frente al no tratamiento de la patología. Williams y coll ${ }^{51}$ concluyen además que la mejoría se asocia a la relación con el terapeuta y la motivación del paciente.

Las investigaciones indican controversias sobre si en el momento de realización de los ejercicios es necesario la supervisión o no de un profesional de los mismos. Felicíssimo y col, ${ }^{22}$ o Zanetti y col ${ }^{53}$ en sus trabajos afirman que esta supervisión no influye en los resultados obtenidos, sin embargo los datos obtenidos en el estudio de Konstantinidou y $\mathrm{col}^{31}$, indican que el grupo que realizó ejercicios de entrenamiento de suelo pélvico bajo supervisión y de forma colectiva obtuvo mejores resultados en el test IQOL, episodios de incontinencia y fuerza muscular vaginal que el grupo que realizó la terapia de forma individualizada en su domicilio y sin supervisión profesional. 
Tabla 2. Calidad metodológica de los estudios incluidos

\begin{tabular}{|c|c|c|c|c|c|c|c|c|c|c|c|c|}
\hline Artículo & 1 & 2 & 3 & 4 & 5 & 6 & 7 & 8 & 9 & 10 & 11 & Puntuación \\
\hline Tejero M y cols. $^{10}$ & Sí & Sí & No & Sí & No & No & No & Sí & No & Sí & Sí & 5. Justo \\
\hline Aksac B y cols. $^{12}$ & No & Sí & Sí & Sí & No & No & No & No & No & Sí & Sí & 4. Justo \\
\hline Alewijnse D y cols. ${ }^{13}$ & Sí & Sí & No & No & No & No & No & No & Sí & Sí & Sí & 4. Justo \\
\hline Arruda R.M y cols. ${ }^{14}$ & No & Sí & No & Sí & No & No & No & No & No & Sí & Sí & 3.Pobre \\
\hline Aukee P y cols..$^{15}$ & Sí & Sí & No & Sí & No & No & No & Sí & Sí & Sí & Sí & 6. Bueno \\
\hline Borello France DF y cols. ${ }^{16}$ & Sí & Sí & Sí & Sí & No & No & No & Sí & Sí & Sí & Sí & 7. Bueno \\
\hline Carneiro EF y cols. ${ }^{17}$ & Sí & Sí & No & Sí & No & No & No & Sí & No & Sí & Sí & 5. Justo \\
\hline Castro RA y cols. ${ }^{18}$ & Sí & Sí & No & Sí & No & No & Sí & Sí & No & Sí & Sí & 6. Bueno \\
\hline De Oliveira Camargo F y cols..$^{19}$ & Sí & Sí & Sí & Sí & No & No & No & Sí & No & Sí & Sí & 6. Bueno \\
\hline Donahoe Fillmore E y cols. ${ }^{20}$ & Sí & Sí & No & No & No & No & No & No & No & Sí & Sí & 3. Pobre \\
\hline Dumoulin $\mathrm{C}$ y cols. ${ }^{21}$ & Sí & Sí & No & Sí & No & No & Sí & Sí & No & Sí & Sí & 6. Bueno \\
\hline FelicísSÍmo MF y cols ${ }^{22}$ & No & Sí & No & Sí & No & No & No & Sí & No & Sí & Sí & 5. Justo \\
\hline Gameiro, MO y cols. ${ }^{23}$ & Sí & No & No & Sí & No & No & No & Sí & No & Sí & Sí & 4. Justo \\
\hline Ghoniem GM y cols. ${ }^{24}$ & Sí & Sí & No & Sí & Sí & No & No & No & Sí & Sí & Sí & 6. Bueno \\
\hline Hirakawa T y cols. ${ }^{25}$ & Sí & Sí & No & Sí & No & No & No & No & Sí & Sí & Sí & 5. Justo \\
\hline Hung HC y cols. ${ }^{26}$ & Sí & Sí & Sí & Sí & No & No & Sí & Sí & Sí & Sí & Sí & 8. Bueno \\
\hline Kamel DM y cols. $^{27}$ & Sí & Sí & Sí & Sí & No & No & No & No & No & No & Sí & 4. Justo \\
\hline Kashanian M y cols. $^{28}$ & Sí & Sí & Sí & Sí & No & No & No & Sí & No & Sí & Sí & 6. Bueno \\
\hline Kim H y cols..$^{29}$ & Sí & Sí & No & Sí & No & No & No & Sí & No & Sí & Sí & 5. Justo \\
\hline Kim H y cols. ${ }^{30}$ & Sí & Sí & No & Sí & No & No & Sí & Sí & No & Sí & Sí & 6. Bueno \\
\hline Konstantinidou E y cols. ${ }^{31}$ & Sí & No & No & Sí & No & No & No & No & No & Sí & Sí & 3. Pobre \\
\hline Liebergall-Wischnitzer M y cols. $^{32}$ & Sí & Sí & No & Sí & No & No & Sí & No & Sí & Sí & Sí & 5. Justo \\
\hline Liebergall-Wischnitzer M y cols. ${ }^{33}$ & Sí & Sí & Sí & Sí & No & No & Sí & No & No & Sí & No & 5. Justo \\
\hline Liebergall-Wischnitzer M y cols. ${ }^{34}$ & Sí & Sí & No & Sí & No & No & Sí & No & No & Sí & Sí & 5. Justo \\
\hline Nascimento-Correia G y cols. ${ }^{35}$ & No & Sí & Sí & No & No & No & No & Sí & No & Sí & Sí & 5. Justo \\
\hline Pereira VS y cols. ${ }^{36}$ & No & Sí & Sí & Sí & No & No & No & Sí & No & Sí & Sí & 6. Bueno \\
\hline Pereira VS y cols. ${ }^{37}$ & Sí & Sí & Sí & No & No & No & No & Sí & Sí & Sí & Sí & 5. Justo \\
\hline Resende AP y cols. ${ }^{38}$ & No & Sí & No & Sí & No & No & Sí & Sí & No & Sí & Sí & 6. Bueno \\
\hline RoongSÍrisangrat S y cols. ${ }^{39}$ & Sí & Sí & No & Sí & No & No & No & Sí & No & Sí & Sí & 5. Justo \\
\hline Sari D; Khorshid L. ${ }^{40}$ & Sí & Sí & No & Sí & No & No & No & No & No & Sí & Sí & 4. Justo \\
\hline Schmidt AP y cols. ${ }^{41}$ & Sí & Sí & No & Sí & No & No & Sí & Sí & No & Sí & Sí & 6. Bueno \\
\hline Schnelle JF y cols. ${ }^{42}$ & Sí & Sí & No & Sí & No & No & Sí & Sí & No & Sí & Sí & 6. Bueno \\
\hline Sherburn M y cols. ${ }^{43}$ & Sí & Sí & Sí & Sí & No & No & Sí & Sí & Sí & Sí & Sí & 8. Bueno \\
\hline Sriboonreung T y cols. ${ }^{44}$ & Sí & Sí & Sí & Sí & No & No & No & Sí & Sí & Sí & Sí & 7. Bueno \\
\hline Sugaya K y cols. ${ }^{45}$ & Sí & Sí & Sí & Sí & Sí & No & No & Sí & No & Sí & Sí & 7. Bueno \\
\hline Tak ECPM y cols. ${ }^{46}$ & Sí & Sí & Sí & Sí & No & No & No & Sí & No & Sí & Sí & 6. Bueno \\
\hline Tang CL y cols. ${ }^{47}$ & Sí & Sí & No & Sí & No & No & No & Sí & No & Sí & Sí & 5. Justo \\
\hline Tsai YC; Liu CH. ${ }^{48}$ & Sí & Sí & No & Sí & No & No & No & Sí & No & Sí & Sí & 5. Justo \\
\hline Vinsnes AG y cols. ${ }^{49}$ & Sí & Sí & Sí & Sí & No & No & No & Sí & No & Sí & Sí & 6. Bueno \\
\hline Wang AC y cols. ${ }^{50}$ & No & Sí & Sí & Sí & No & No & No & Sí & No & Sí & Sí & 5. Justo \\
\hline Williams KS y cols ${ }^{51}$ & Sí & Sí & Sí & Sí & No & No & No & Sí & Sí & Sí & Sí & 7. Bueno \\
\hline Yoon HS y cols. ${ }^{52}$ & Sí & Sí & No & Sí & No & No & Sí & Sí & No & Sí & Sí & 6. Bueno \\
\hline Zanetti MR y cols. ${ }^{53}$ & No & Sí & No & Sí & No & No & No & No & No & Sí & No & 3. Pobre \\
\hline
\end{tabular}

Sí: Presencia del criterio; NO: No presencia del criterio. 
Sería necesario poder comparar los resultados obtenidos con y sin supervisión realizando ambos grupos los ejercicios de forma individualizada, ya que en base a la bibliografía consultada los mejores resultados obtenidos por Konstantinidou y $\mathrm{col}^{31}$ podrían deberse a la motivación que proporciona el trabajo en grupo más que a la supervisión de un profesional ${ }^{19,37}$.

La primera línea de tratamiento utilizada para tratar la IUE o IUM es un programa de entrenamiento de la musculatura del suelo pélvico ${ }^{22}$, además la evidencia médica muestra que el entrenamiento de la musculatura del suelo pélvico obtiene mejores resultados como método de tratamiento de la IUE o IUM que algunos fármacos utilizados comúnmente para el tratamiento de dicha patología como son la oxibutinina ${ }^{14}$ o duloxetina ${ }^{23}$. Actualmente la oxibutinina ha dejado de usarse a favor de antimuscarínicos como fesoterodina, tolterodina o solifenacina ${ }^{54}$. Por otro lado, duloxetina, a pesar de estar indicada para IUE, no está siendo administrada de forma rutinaria dados sus escasos resultados ${ }^{54}$.

En base a estas conclusiones podrían considerarse los ejercicios de suelo pélvico como método alternativo o complementario a la toma de fármacos por las pacientes afectas, reduciendo de esta manera los efectos secundarios que se derivan de estos medicamentos.

Hay que tener en cuenta que la UI es una enfermedad crónica que afecta, de manera importante, a la calidad de vida, por lo que sería necesario evaluarla en los pacientes afectos mediante cuestionarios validados, ya que una reducción de los episodios de perdida orina o un incremento en el tono muscular no siempre se traduce en una mejora de la calidad de vida. Sería conveniente realizar un seguimiento de los pacientes a largo plazo incluyendo este parámetro.

Las conclusiones de este estudio se han realizado tomando como base los artículos localizados por la estrategia de búsqueda empleada y por la selección con los criterios de inclusión y exclusión propuestos. Aun así, se debe de considerar la probabilidad de que existan estudios que por problemas de indexación o límites de búsqueda hayan quedado fuera de esta revisión.

\section{CONSIDERACIONES FINALES}

En base a los estudios publicados, el tratamiento de la incontinencia urinaria de esfuerzo o mixta mediante ejercicios de entrenamiento de la musculatura del suelo pélvico contribuye de manera positiva a mejorar los síntomas, produciendo mejoras significativas en la fuerza de la musculatura del suelo pélvico, calidad de vida de las pacientes afectas y reducción del número de episodios de incontinencia.

Sería necesario un mayor número de estudios para determinar de forma concreta el tipo de ejercicio y duración del mismo más adecuado a cada tipo de incontinencia.

\section{BIBLIOGRAFÍA}

1. Estudillo F, Garrido S, Sánchez C, Nieto M, RoDRíGUEZ F. Incontinencia urinaria en la mujer ¿qué debemos saber? Enfuro 2004; 91: 10-13.

2. MilLA F.J. Protocolo de reeducación vesical en incontinencia urinaria. Enfuro 2007; 102: 14-17.

3. ENRIQUE J. La incontinencia urinaria. An Sist Sanit Navar 2006; 29: 219-231

4. SÁnchez M, Vázquez E, López MC, Rodríguez MC. Incontinencia urinaria y mujer: un problema frecuente y olvidado. Med Gen 2006; 91: 790793.

5. Senovilla JL, Guinda A, Gimeno A, Zazo J, Borrego A, García A. Conocimientos básicos sobre incontinencia urinaria femenina de esfuerzo. Clín Urol Complut 2000; 8: 377-394.

6. Delgado H, Ramírez JM, Pereda E, Delgado MJ. Detección de incontinencia urinaria. Enfuro 2007; 102: 10-13.

7. Juarranz M, Terron M, Roca M, Soriano T, VillaMOR M, CALVo MJ. Tratamiento de la incontinencia urinaria. Aten Primaria 2002; 30: 323332.

8. Petros Pe, Ulmsten UI. An integral theory of female urinary incontinence. Experimental and clinical considerations. Acta Obstet Gynecol Scand Suppl 1990; 153: 7-31.

9. Petros PE, Ulmsten UI. An integral theory and its method for the diagnosis and management of female urinary incontinence. Scand J Urol Nephrol Suppl 1993; 153: 1-93. 
10. Tejero M, Marco E, Boza R, Selva F, Piqueras M, GuLLÉN A y col. Incontinencia urinaria de esfuerzo y ejercicios del suelo pélvico. Estudio comparativo entre la eficacia de un entrenamiento individualizado vs instrucciones básicas. Trauma 2008; 19: 171-177.

11. Escala Pedro [Consultado 01/06/2013]. Disponible en: http://www.pedro.org.au/spanish/ downloads/pedro-scale/.

12. Aksac B, Aki S, Karan A, Yalcin O, Isikoglu M, ESKIYURT N. Biofeedback and pelvic floor exercises for the rehabilitation of urinary stress incontinence. Gynecol Obstet Invest 2003; 56: 23-27.

13. Alewijnse D, Metsemakers JF, Mesters IE, Van den BORNE B. Effectiveness of pelvic floor muscle exercise therapy supplemented with a health education program to promote longterm adherence among women with urinary incontinence. Neurourol Urodyn 2003; 22: 284-295.

14. Arruda RM, Castro RA, Sousa GC, Sartori MG, BARACAT EC, GiRÃo MJ. Prospective randomized comparison of oxybutynin, functional electrostimulation, and pelvic floor training for treatment of detrusor overactivity in women. Int Urogynecol J Pelvic Floor Dysfunct 2008; 19: 1055-1061.

15. Aukee P, Immonen P, LaAksonen DE, Laippala P, Penttinen J, Airaksinen O. The effect of home biofeedback training on stress incontinence. Acta Obstet Gynecol Scand 2004; 83: 973-977.

16. Borello DF, Downey PA, Zyczynski HM, Rause CR. Continence and Quality of Life in Female Stress Urinary Incontinence. Phys Ther 2008; 88: 1545-1553.

17. Carneiro EF, Araujo Ndos S, Beuttenmüll L, VieiRA PC, CADER SA, CADER SA y col. The anatomical-functional characteristics of the pelvic floor and quality of life of women with stress urinary incontinence subjected to perineal exercises. Actas Urol Esp 2010; 34: 788-793.

18. Castro RA, Arruda RM, Zanetti MR, Santos PD, SARTORI MG, GIRÃo MJ. Single-blind, randomized, controlled trial of pelvic floor muscle training. Clinics 2008; 63: 465-472.

19. De Oliveira F, Rodrigues AM, Arruda RM, FerreiRA MG, Girão MJ, Castro RA. Pelvic floor muscle training in female stress urinary incontinence: comparison between group training and individual treatment using PERFECT assessment scheme. Int Urogynecol J Pelvic Floor Dysfunct 2009; 20: 1455-1562.

20. Donahoe DE, Chorny W, Brahler CJ, Ingley A, Kennedy J, Osterfeld V. A comparison of two pelvic floor muscle trainings programs in female with stress urinary incontinence: a pilot study. J Appl Res 2011; 11: 73-83.

21. Dumoulin C, Lemieux MC, Bourbonnais D, Gravel D, Bravo G, Morin M. Physiotherapy for persistent postnatal stress urinary incontinence: a randomized controlled trial. Obstet Gynecol 2004; 104: 504-510.

22. Felicíssimo MF, Carneiro MM, Saleme CS, Pinto RZ, DA Fonseca AM, DA Silva-Filho AL. Intensive supervised versus unsupervised pelvic floor muscle training for the treatment of stress urinary incontinence: a randomized comparative trial. Int Urogynecol J 2010; 21: 835-840.

23. Gameiro MO, Moreira EH, Gameiro FO, Moreno JC, Padovani CR, Amaro JL. Vaginal weight cone versus assisted pelvic floor muscle training in the treatment of female urinary incontinence. A prospective, single-blind, randomized trial. Int Urogynecol J 2010; 21 : 395-399.

24. Ghoniem GM, Van LeEuwen JS, Elser DM, FreeMAN RM, ZHAO YD, YALCIN I y col. A randomized controlled trial of duloxetine alone, pelvic floor muscle training alone, combined treatment and no active treatment in women with stress urinary incontinence. J Urol 2005; 173: 1647-1653.

25. Hirakawa T, Suzuki S, Kato K, Gotoh M, YosHIKAWA Y. RANDOMIZED controlled trial of pelvic floor muscle training with or without biofeedback for urinary incontinence. Int Urogynecol J 2013; 24: 1347-1354.

26. Hung HC, Hsiao SM, Chin SY, Lin HH, Tsauo JY. An alternative intervention for urinary incontinence: retraining diaphragmatic, deep abdominal and pelvic floor muscle coordinated function. Man Ther 2010; 15: 273-279.

27. Kamel DM, Thabet A, Tantawy S, Radwan M. Effect of abdominal versus pelvic floor muscle exercises in obese Egyptian women with mild stress urinary incontinence: A randomized controlled trial. HKPJ 2013; 31: 12-18.

28. Kashanian M, Ali SS, Nazemi M, Bahasadri S. Evaluation of the effect of pelvic floor muscle training (PFMT or Kegel exercise) and assisted pelvic floor muscle training (APFMT) by a resistance device (Kegelmaster device) on the urinary incontinence in women: a randomized trial. Eur J Obstet Gynecol Reprod Biol 2011; 159: 218-223.

29. Kim H, Suzuki T, Yoshida Y, Yoshida H. Effectiveness of multidimensional exercises for the treatment of stress urinary incontinence in elderly community-dwelling Japanese women: a randomized, controlled, crossover trial. J Am Geriatr Soc 2007; 55: 1932-1939. 
30. Kim H, Yoshida H; Suzuki T. The effects of multidimensional exercise treatment on community-dwelling elderly Japanese women with stress, urge, and mixed urinary incontinence: a randomized controlled trial. Int J Nurs Stud 2011; 48: 1165-1172.

31. Konstantinidou E, Apostolidis A, Kondelidis N, Tsimtsiou Z, Hatzichristou D, IoAnnides E. Shortterm efficacy of group pelvic floor training under intensive supervision versus unsupervised home training for female stress urinary incontinence: a randomized pilot study. Neurourol Urodyn 2007; 26: 486-491.

32. Liebergall M, Hochner D, Lavy Y, Manor O, Arbel R, Paltiel O. Paula. Method of circular muscle exercises for urinary stress incontinence: a clinical trial. Int. Urogynecol. J Pelvic Floor Dysfunct. 2005; 16: 345-351.

33. Liebergall M, Hochner D, Lavy Y, Manor O, ShVeiky D, Paltiel O. Randomized trial of circular muscle versus pelvic floor training for stress urinary incontinence in women. J. Womens Health 2009; 18: 377-385.

34. Liebergall M, Paltiel O, Hochner D, Lavy Y, Manor O, Woloski AC. Sexual function and quality of life of women with stress urinary incontinence: a randomized controlled trial comparing the Paula method (circular muscle exercises) to pelvic floor muscle training (PFMT) exercises. J Sex Med 2012; 9: 16131623.

35. Nascimento G, Santos V, Tahara N, Driusso P. Effects of pelvic floor muscle training on quality of life of a group of women with urinary incontinence: randomized controlled trial. Actas Urol Esp 2012; 36: 216-221.

36. Pereira VS, Correia GN, Driusso P. Individual and group pelvic floor muscle training versus no treatment in female stress urinary incontinence: a randomized controlled pilot study. J Obstet. Gynecol Reprod Biol 2011; 159: 465-471.

37. Pereira VS, De Melo MV, Correia GN, Driusso P. Vaginal cone for postmenopausal women with stress urinary incontinence: randomized, controlled trial. Climacteric 2012; 15: 4551.

38. Resende AP, Stüpp L, Bernardes BT, Oliveira E, Castro RA, Girão MJ. Can hypopressive exercises provide additional benefits to pelvic floor muscle training in women with pelvic organ prolapse? Neurourol Urodyn 2012; 31: 121-125.

39. Roongsirisangrat S, Rangkla $\mathrm{S}$, Manchana T, TanTISIRIWAT N. Rectal balloon training as an adjunctive method for pelvic floor muscle tra- ining in conservative management of stress urinary incontinence: a pilot study. J Med Assoc Thai 2012; 95: 1149-1155.

40. SARI D, KHORSHID L. The effects of pelvic floor muscle training on stress and mixed urinary incontinence and quality of life. J. Wound Ostomy Continence Nurs 2009; 36: 429-435.

41. Schmidt AP, Sanches PR, Silva DP JR, Ramos JG, Nohama P. A new pelvic muscle trainer for the treatment of urinary incontinence. Int $\mathrm{J}$ Gynaecol Obstet 2009; 105: 218-222.

42. Schnelle JF, Kapur K, Alessi C, Osterweil D, Beck JG, Al-Samarrai NR y col. Does an exercise and incontinence intervention save healthcare costs in a nursing home population? J Am Geriatr Soc 2003; 51: 161-168.

43. Sherburn M, Bird M, Carey M, Bø K, Galea MP. Incontinence improves in older women after intensive pelvic floor muscle training: an assessor-blinded randomized controlled trial. Neurourol Urodyn 2011; 30: 317-324.

44. SRiboonreung T, Wongtra-ngan S, EunginichPong W, LaOpaiBoon M. Effectiveness of pelvic floor muscle training in incontinent women at Maharaj Nakorn Chiang Mai Hospital: a randomized controlled trial. J Med Assoc Thai 2011; 94: 1-7.

45. Sugaya K, Owan T, Hatano T, Nishijima S, Miyazato M, Mukouyama H y col. Device to promote pelvic floor muscle training for stress incontinence. Int J Urol 2003; 10: 416-422.

46. TaK E, Van Hespen, A, Van Dommelen P, Hopman M. Does improved functional performance help to reduce urinary incontinence in institutionalized older women? A multicenter randomized clinical trial. BMC Geriatrics 2012; 12-51.

47. TANG CL, Dai DC, ZHU WF, Jin YY, Mei LF, ZhaO GF. Observation on therapeutic effect of dog-day acupuncture and moxibustion combined with pelvic floor muscle exercises for treatment of female stress urinary. Zhongguo Zhen Jiu 2009; 29: 879-883.

48. Tsai YC, LIU CH. The effectiveness of pelvic floor exercises, digital vaginal palpation and interpersonal support on stress urinary incontinence: an experimental study. Int J Nurs Stud 2009; 46: 1181-1186.

49. Vinsnes AG, Helbostad JL, Nyrønning S, Harkless GE, GRanbo R, SEIm A. Effect of physical training on urinary incontinence. Clin Interv Aging 2012; 7: 45-50.

50. Wang AC, Wang YY, Chen MC. Single-blind, randomized trial of pelvic floor muscle training, biofeedback-assisted pelvic floor muscle training, and electrical stimulation in the management of overactive bladder. Urology 2004; 63: 61-66. 
51. Williams K.S, Assassa RP, Gillies CL, Abrams KR, Turner DA, SHAW C y col. A randomized controlled trial of the effectiveness of pelvic floor therapies for urodynamic stress and mixed incontinence. BJU Int 2006; 98: 1043-1050.

52. Yoon HS, Song HH, Ro YJ. A comparison of effectiveness of bladder training and pelvic muscle exercise on female urinary incontinence. Int J Nurs Stud 2003; 40: 45-50.
53. Zanetti MR, Castro Rde A, Rotta AL, Santos PD, SARTORI M, GIRÃo MJ. Impact of supervised physiotherapeutic pelvic floor exercises for treating female stress urinary incontinence. Sao Paulo Med J 2007; 125: 265-269.

54. Shamliyan T, Wyman J, Kane RL. Non surgical treatments for urinary incontinence in adult women: diagnosis and comparative effectiveness. Comparative Effectiveness Review $2012 ; 36$. 